$17^{\text {th }}$ International Congress of Metrology, 02014 (2015)

DOI: $10.1051 /$ metrology / 201502014

(C) Owned by the authors, published by EDP Sciences, 2015

\title{
Etude statistique de la dérive du pied à coulisse
}

\author{
Christophe DUBOIS ${ }^{1}$ \\ ${ }^{1}$ Delta Mu, Centre d'affaire du Zénith, Le trident E - 48 rue Sarliève,63800 Cournon d'Auvergne
}

\begin{abstract}
Knowing the drift of a caliper, one might be able to determine the right frequency calibration that should be attributed to it. However, when trying to learn about the order of such a drift, opinion is divided on the question. Some metrologists say that the caliper does not drift while others claim to have established a periodicity according to an observed drift. From real calibrations of over a hundred calipers, this study tries to prove the drift or not of calipers in use in the industrial world.
\end{abstract}

\section{Introduction}

Certains métrologues affirment que les pieds à coulisse (PAC) ne dérivent pas alors que d'autres affirment avoir établi une périodicité d'étalonnage en fonction d'une dérive constatée. A partir de l'étude de plusieurs centaines d'étalonnage de PAC, cet article essaie d'étudier la dérive (ou non-dérive) des PAC.

\section{Définition de la dérive}

Avant d'aller plus loin, il faut d'abord se mettre d'accord sur le terme " dérive ». Le fait de constater, par exemple, que $5 \%$ des PAC reviennent chaque année « non conformes » ne démontre pas une dérive du moyen. Il faut séparer les résultats issus d'un étalonnage périodique qui représentent l'évolution " naturelle » du moyen, des étalonnages " évènementiels » qui interviennent suite à un doute, un choc ou une chute du moyen donc suite à un comportement atypique sur le moyen. Dans ce second cas, on ne peut pas parler de dérive.

De plus il faut aussi bien définir le sens du terme dérive. Le Vocabulaire international de la métrologie (VIM)[1] le définit de la manière suivante :

\subsection{1 dérive instrumentale}

Variation continue ou incrémentale dans le temps

d'une indication, due à des variations des propriétés métrologiques d'un instrument de mesure.

Cependant, malgré l'existence de cette définition, son interprétation est toujours possible. Dans le cas de l'étalonnage je l'interpréterais de la façon suivante :

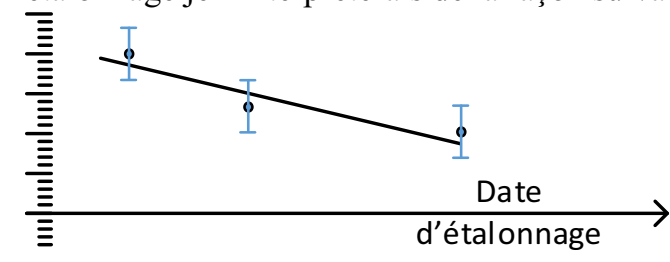

Email de correspondance de l'auteur : cdubois@deltamu.fr
Figure 1 : dérive en étalonnage

En revanche, pour un appareil qui présente des écarts aléatoires à chaque étalonnage, suffisamment importants pour être ajustés, il me parait impropre d'utiliser le terme « dérive ». En effet les variations ne sont pas incrémentales dans le temps, parfois elles sont au-dessus ou en dessous de la valeur de référence.

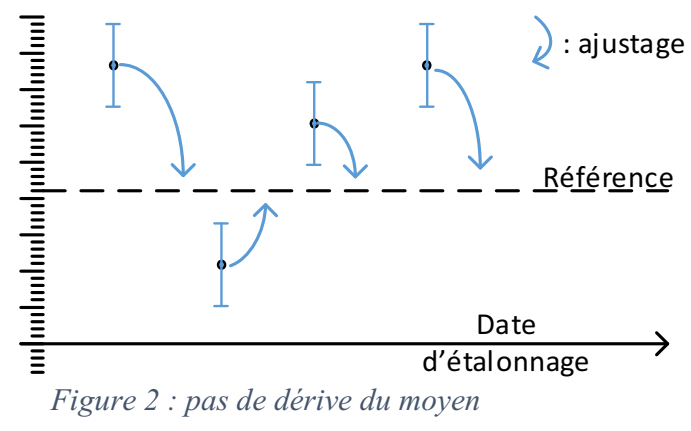

Dans ce cas, l'appareil se dérègle mais le terme de dérive n'est pas approprié et peut même dans certains cas prêter à confusion. En utilisant le terme dérive pour ce genre de comportement, on laisse croire que l'écart constaté va s'aggraver dans le temps. Ce qui est faux car le sens de variation et la raison de la variation ne sont pas connus (pas de variation continue ou incrémentale dans le temps). Le moyen peut rester longtemps dans cet état voir changer de sens de variation. Il est donc important de bien dissocier les 2 comportements afin d'y apporter les solutions adaptées. Par exemple dans le cas du choix de la périodicité d'étalonnage, il est possible de faire une étude statistique de la dérive pour le premier cas et une étude statique de fiabilité dans le second cas. 


\section{Principe de l'étude}

Le but de l'article est d'étudier la dérive du PAC selon mon interprétation de la définition du VIM. Est-ce que les PAC s'usent (dérivent) au cours du temps (comme dans la Figure 1) et dans quel sens, ou bien est-ce qu'ils se dérèglent de manière aléatoire pour des raisons ponctuelles (chute, coup, ...) non incrémentales (comme dans la Figure 2). Les données de l'étude sont issues de PV d'étalonnage de pieds à coulisse.

\subsection{Paramètre étudié et problématique}

L'article se limite à l'étude de l'erreur d'indication pleine cale des becs principaux. À partir des données de 2 étalonnages successifs, une pente d'usure est estimée pour chaque PAC en faisant la différence des erreurs entre les 2 dates d'étalonnage par rapport au temps écoulé.

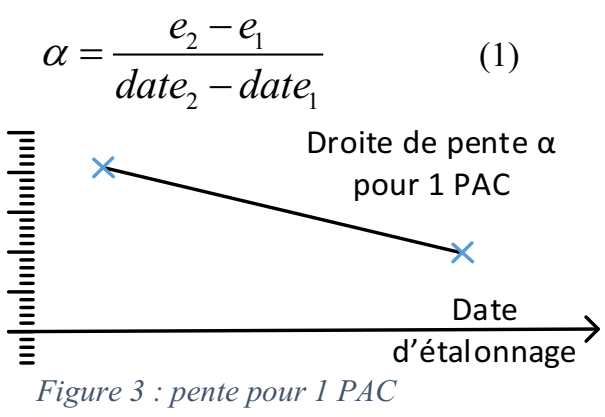

À partir d'un grand nombre de pentes, il devient possible d'étudier une pente moyenne qui correspond à une usure moyenne des PAC.

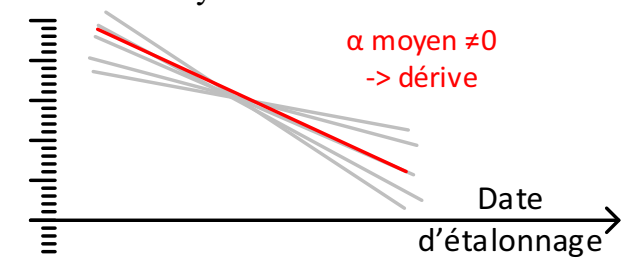

Figure 4: cas où la pente moyenne montre une dérive

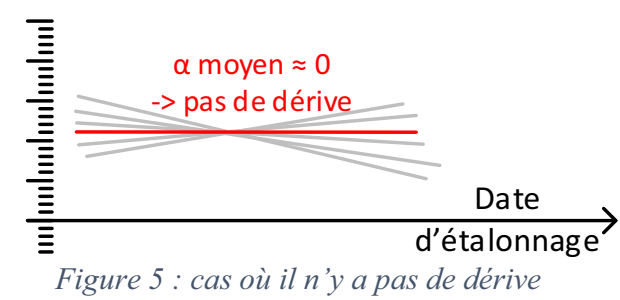

Cependant, afin de réellement conclure à une dérive, il faut connaitre les caractéristiques de la loi de distribution des pentes. C'est donc avec des représentations de loi de distribution que le raisonnement sera fait.

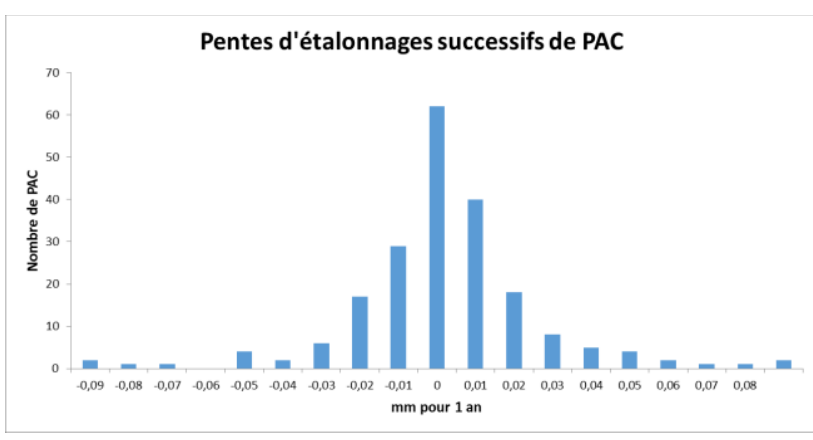

Figure 6 : Densité de probabilité des pentes

\subsection{Les sources des PV d'étalonnages réels}

Les résultats de l'étude dépendent évidemment de la nature des données utilisées. L'étude porte sur des cas réels de 658 étalonnages issus de $225 \mathrm{PAC}$ (pouvant avoir été étalonnés plusieurs fois) provenant de 8 entreprises dans le domaine de la mécanique. Les étalonnages sont en majorité effectués par des laboratoires COFRAC dans le domaine. Seulement 148 PAC présentent au moins 2 étalonnages. En tout, 213 pentes sont étudiées.

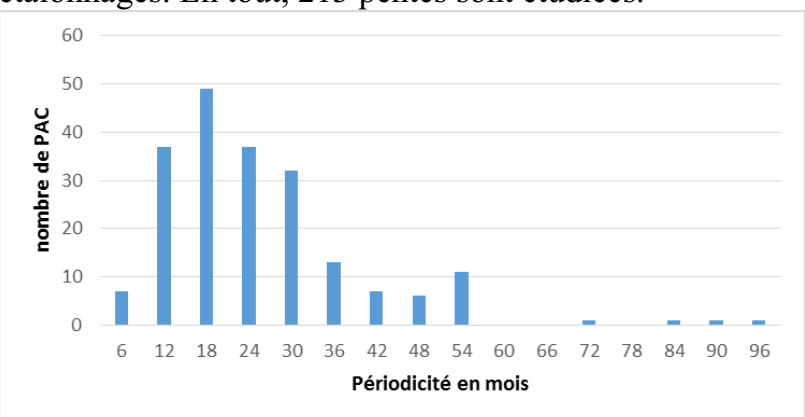

Figure 7 : Répartition de dates entre 2 étalonnages

\subsection{Modélisation d'étalonnage de PAC parfait}

Les résultats réels sont ensuite comparés à des résultats d'étalonnage modélisés de PAC parfaits (c'est à dire sans dérive) étalonnés avec la même périodicité et en tenant compte des incertitudes annoncées des laboratoires d'étalonnage. Si la modélisation se superpose à la courbe réelle cela signifie que la réalité se comporte comme des $\mathrm{PAC}$ sans dérive.

\section{Analyse des valeurs}

\subsection{Cas réelles}

Sur les 213 pentes, 8 valeurs ont été écartées pour les raisons suivantes :

- Etalonnage successif dans une période inférieure à 4 mois pour $7 \mathrm{PAC}$. Le résultat est jugé non significatif car trop dépendant de l'incertitude d'étalonnage

- 1 valeur aberrante. Ecart de -3 mm entre deux étalonnages espacés de 9 mois. Il s'agit soit d'une erreur de mesure, soit d'une chute ou d'un coup reçu pas le PAC et en aucun cas d'une dérive. 
Les 205 résultats sont présentés dans la figure suivante. Les pentes sont exprimées en mm/an.

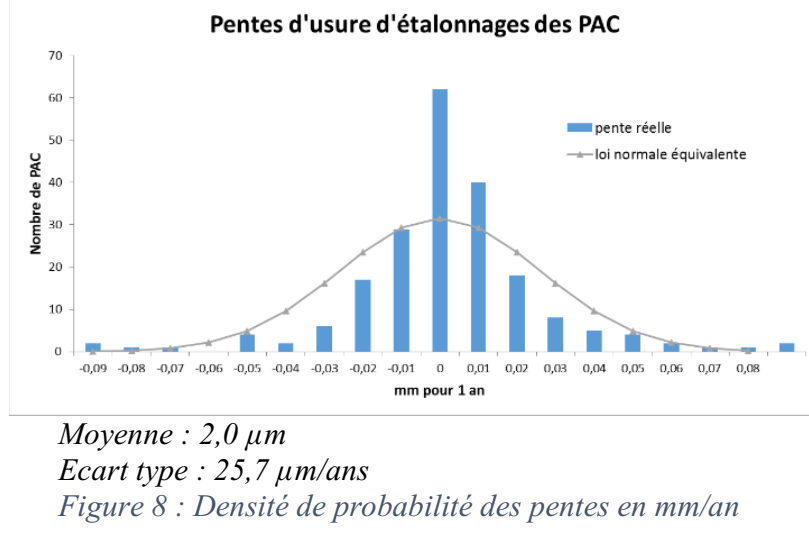

Sur ce premier résultat, on constate que le graphique semble symétrique et centré sur 0 (dérive nulle). Les dispersions des résultats sont de l'ordre de grandeur des incertitudes d'étalonnage annoncées par les laboratoires. L'ensemble de ces constatations semble infirmer la présence d'une dérive, ou alors très faible par rapport aux incertitudes d'étalonnage.

La distribution est « trop pointue » pour être assimilée à une loi normale comme le confirme la droite de Henry Figure 9.

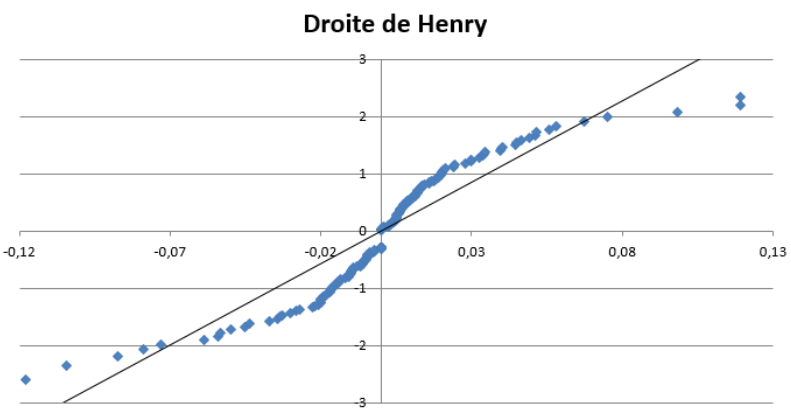

Figure 9: Droite de Henry des pentes

Cela limite l'utilisation de tests statistiques classiques pour juger des valeurs de la courbe, notamment la significativité du 0 .

\section{2 Étalonnage de PAC parfaits}

L'idée est de comparer le résultat réel (précédent) avec le résultat que l'on pourrait obtenir avec des PAC ne dérivant pas (qualifié de « parfaits » pour l'étude).

Il est possible de déterminer le comportement d'une famille de PAC ne dérivant pas, donc de pente moyenne nulle, étalonnés avec la même fréquence que la famille réelle. La pente est déterminée à partir de 2 paramètres, d'une part les écarts, d'autre part le temps entre les étalonnages. Si les étalonnages sont réalisés à des dates très éloignées, la variation des pentes possibles est faible, complètement amortie par le temps entre 2 étalonnages.
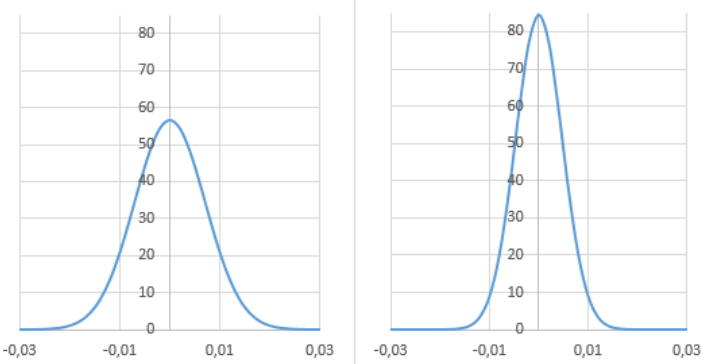

Figure 10 : distribution des pentes pour deux PAC parfaits, de périodicité 12 mois et 18 mois

Il est donc important de reconstituer la famille de PAC parfaits avec les mêmes fréquences que la famille de référence réelle.

La pente est supposée distribuée selon une loi normale car issue de la différence de 2 étalonnages distribués euxmêmes selon une loi normale. L'incertitude sur la pente se déduit par la loi de propagation à partir de l'équation (1) :

$$
u_{\text {pente }}=\frac{\sqrt{2} u_{\text {étalonnage }}}{\text { périodicité }}
$$

Pour chaque PAC de la famille, la densité de probabilité est déduite en fonction de la périodicité. Les étalonnages sont supposés indépendants. La densité de probabilité de la famille totale est déduite par la somme des densités de probabilité.

L'incertitude d'étalonnage annoncée par les différents laboratoires varie entre $20 \mu \mathrm{m}$ et $25 \mu \mathrm{m}$. L'incertitude moyenne des différents étalonnages réels de l'étude est de $22 \mu \mathrm{m}$. Donc une incertitude type moyenne de $11 \mu \mathrm{m} / \mathrm{an}$ est utilisée pour l'étude.

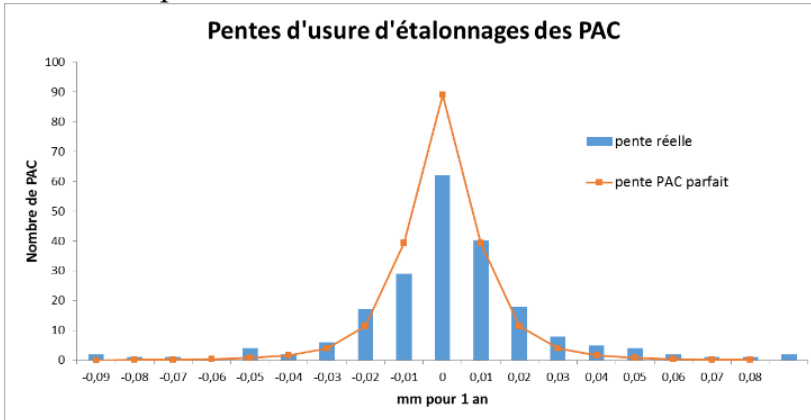

Figure 11 : Pentes PAC parfaits vs PAC réels

\subsection{Comparaisons des courbes}

Les 2 courbes sont proches. La modélisation d'étalonnage de PAC parfaits (sans dérive) est donc comparable aux résultats des PAC réels. Ce qui semble aller dans le sens que les PAC ne dérivent pas.

On constate tout de même un certain écart entre les 2 courbes.

La modélisation est plus pointue que la réalité, donc la dispersion réelle est légèrement plus importante. Plusieurs hypothèses peuvent expliquer cette dispersion.

- une modélisation trop restrictive sur les incertitudes choisies. Avec une incertitude type de $16 \mu \mathrm{m} / \mathrm{an}$ (au lieu de $11 \mu \mathrm{m} / \mathrm{an}$ ), les courbes se superposent parfaitement. Mais cela correspond à une incertitude d'étalonnage de $32 \mu \mathrm{m}$, ce qui me parait un peu excessif. 
- des déréglages réels des PAC de quelques microns. Ces déréglages sont visibles en moyenne sur la dispersion des résultats de la famille, mais ils sont difficilement détectables sur un seul résultat d'étalonnage étant données les incertitudes d'étalonnages. Cette dispersion supplémentaire est assimilable à une dispersion de $12 \mu \mathrm{m} / \mathrm{an}$ pour arriver au $16 \mu \mathrm{m} / \mathrm{an} \mathrm{du}$ paragraphe précédent $\left(12 \mu \mathrm{m} / \mathrm{an}=\right.$ racine $\left.\left(16^{2} \mu \mathrm{m} / \mathrm{an}-11^{2} \mu \mathrm{m} / \mathrm{an}\right)\right)$. Mais attention, il ne s'agit pas d'une dérive de $12 \mu \mathrm{m} / \mathrm{an}$ comme pourrait le laisser penser les unités, c'est simplement une modélisation des déréglages réels constatés, normalisés sur 1 année. Si l'échantillon de l'étude avait par exemple une périodicité plus importante mais des résultats d'étalonnages similaires (ce qui est probable étant donnée la supposition de non dérive des PAC), les dispersions des pentes auraient été plus faibles.

Mais dans tous les cas, ces écarts qui accroissent la dispersion des résultats, ne peuvent pas être qualifiés de dérive. En effet, ils sont présents de chaque côté de la courbe. Il ne s'agit donc pas d'une «variation continue ou incrémentale dans le temps ».

La courbe réelle semble également légèrement décalée vers la droite. Il peut effectivement s'agir d'un phénomène de dérive moyenne des PAC. Mais le décalage, s'il est réellement avéré, est autour de $2 \mu \mathrm{m}$ par an, il ne peut être constaté lors d'un étalonnage unique et encore moins lors d'une mesure avec le PAC en condition d'utilisation.

En conclusion, cette étude montre que sur l'échantillon de PAC étudiés, aucune dérive significative n'a pu être constatée. On peut alors s'interroger sur l'intérêt de l'étalonnage périodique de ce type de moyen. Une surveillance du moyen dans son milieu d'utilisation parait mieux adaptée à la réalité de son comportement.

\section{Perspectives}

La présente étude peut être approfondie en prenant en compte les éléments suivants :

- La modélisation est basée sur une incertitude moyenne de $22 \mu \mathrm{m}$ par PAC. Afin d'affiner les résultats, il est possible de prendre l'incertitude annoncée pour chaque PAC et non pas la moyenne. Cependant, les laboratoires travaillant de manière similaire, les différences annoncées ne représentent peut-être pas une réalité.

- Les résultats d'étalonnage sont en fait le maximum de 5 mesures. Ce paramètre n'a pas été pris en compte dans la modélisation. Intuitivement, il aurait tendance à élargir la dispersion de la modélisation.

- Compléter la modélisation en introduisant des déréglages sur les PAC pour s'approcher de la courbe réelle et ainsi caractériser les déréglages possibles.

- Vérifier que la dérive constatée autour de $2 \mu \mathrm{m} / \mathrm{an}$ est avérée et non pas un effet statistique.
Mais l'intérêt de l'étude est d'identifier le comportement du moyen dans le temps afin d'ajuster au mieux une surveillance. Dans le cas des PAC, il semble que l'étalonnage périodique ne puisse pas réellement détecter de problèmes. Et si aussi peu de PAC dans l'échantillon étudié montrent d'écart significatifs par rapport à l'incertitude d'étalonnage, c'est que lorsque le problème survient, il est rapidement identifié sans étalonnage (bec tordus, jeux important,...). Le PAC est un moyen robuste, il ne dérive pas mais prend des coups ou subit des chutes qui sont soit sans conséquences, soit très rapidement identifiés.

Ce type d'analyse peut être reproduit pour tout autre moyen pour lequel il existe un doute sur la dérive réel, et ainsi justifier de stratégies mieux adaptées.

\section{Références}

1. Vocabulaire international de métrologie Concepts fondamentaux et généraux et termes associés (VIM) JCGM 200 (Joint Committee for Guides in Metrology), version 2008 